\title{
Sebastian Scheerer Neue soziale Bewegungen und Strafrecht
}

Zur Umschreibung der heterogenen Protestbewegungen, gegenkulturellen Strömungen, Bürgerinidariven und Selbschilfeprojekte, die sich mit und seit der Studentenbewegung in den encwickelten kapitalisrischen Ländern herausbildeten, hat sich das Wort von den «neuen sozialen Bewegungen e etabliert. Zu ihnen zählen Altemarivbetriebe wie Anti-Kernkraft-Initiativen, Instandbesęzer und Ökologen, die Fraven- und die neue Friedensbewegung, abweichende Lebensstilszenen und sexuelle Minderheiten ebenso wie regionalistische Bewegungen. Bei aller UnterschiedLichkeic nach Zielen, Organisations- und Akrionsformen markieren sie doch gemeinsam adas flüssige Element im Gegensarz zu den formalisierten politischen Serukturen, spontane Organisationsbildungen in einer bürokratisierten Welta (Roth 1982, 79). Wrll man sich der Geschichte der neuen sozialen Bewegungen in periodisierender Absicht nähern, so bietet sich an, eine "Phasen-" Oder "Stufen-x Einteilung anhand der sich verändernden Haltung zu Recht und Staat zu probieren. Das Resultac wäre dann vielleicht eine Dreigliederung dieser Art:

In der ersten Phase (späte sechziger Jahre) war ibr Selbstverständnis liberrär, antünsticucionell, akephal und antietatistisch. In der zweiten (frühe siebziger Jahre) war zwar die Schubkraft der Revolte verlorengegangen, doch man sammelte sich in breiter Front auf der Grundwelle eines viel umfassenderen Dissenses zu herrschenden Werten und Normen - und entwickelte einen Anerkennungs-Anspruch den Insaiturionen gegenüber. So wie Tamar Pirch $(1983)$ es in bezug auf die italienische Frauenbewegung beschreibt, war es niche nur dort: In den Akcionen und Forderungen rückten „Rechtsgleichheiz* und „volle Bürgerrechte* gegenüber der antiinstitutionellen Betonung von prinzipieller Differenz in den Vordergrund; das Repräsenrationsprinzip erlangte immer mehr Anerkennung, was wiederum die Mitarbeit in Institutionen und deren Legitimation erleichtere. Was aber am wichtigsten war: Das Strafrecht, einst Inkarmacion nichs nur falscher Inhalte, sondern auch eines falschen Prinzips, erlangte im Verlauf der sicbziger Jahre wieder Anerkennung als eines der bedeutendsten symbolischen Mitrel zur Erablierung allgemeiner Prinzipien. Die [dee einer durch positive Rechrsänderungen zu erreichenden "Gerechrigkeit " auf der Basis des Schuldprinzips gewann neue Anhänger. Ist dem Strafrecht aber erst einmal die Rolle eines universełlen symbolischen Organisators der Hierarchie von Werten und Gütern zugewiesen, dann ist schon die dritte Phase der Blickverengung auf die Parcizipation in den bestehenden Institutionen (späte siebziger, frühe achcziger Jahue) erreicht. Die sozialen Bewegungen wollen dern Staat nichis mehr tun, werin er nur verspricht, mehr für sie zu run.

In den Augen der neuen sozialen Bewegungen gewinnt das Sirafrecter damit die verlorene quasi-metaphysische Würde zurück. Es wird zum Mitrel der symbolischen Fesuschreibung der neuen Wertordnung. Ob das Gesetz im technischen Sinn weffekciv o oder gar "kontraprodukuv* ist, erscheint den neuen Kriminalisierern 
nicht mehr so wichtig: Hauptsache, es ist ihr Gesetz. Weniger die Konfliktregelung als die Statusdarstellung ist Aufgabe der neuen Strafgeserzgebung. Die Folge: eine Kriminalisierungswelle in den Bereichen der Moral- und Kommunikationsdelikte, dort also, wo Kritiker noch vor einem Jahrzehnt die Zweckmäßigkeit und Zulässigkeit staatlich-strafrechtlicher Regelung überhaupt in Frage gestellt hatten.

War die dominierende Tendenz bis in die siebziger Jahre die negacive Kritik am Scrafrecht als eines staaclichen Machtinstruments überhaupt und suchte man folglich nach Alternativen zum Strafrecht (vgl. Steiner 1985), so führte der etacistische Umschlag der wichrigsten der neuen Bewegungen zum nachhalrigen Einklagen a'ternativer Inhalte im Strafrecht. Zu einem Zeirpunkt, an dern die sozialen Bewegungen aus dem sozialen und politischen Alltag nicht mehr wegzudenken sind und mehr Menschen aktivieren und stärkeren Einfluß auf die Inhalte der politischen Konflikte nehmen als die alten Parteien (vgl. Roch 1982, a.a. O.), mausem sich die ehernals schärfsten Kritiker der strafrechtlichen Repressionsinstrumente zum einflußreichen Strafgeserzgeber im materiellen Sinne. Gewiß: Im politischen Streit um die Ausstaatung einer neuen Moral mic den Insignien repressiver Allgemeinverbindlicbkeic werden die neuen sozialen Bewegungen nichr irnmer obsiegen. Doch schon der Versuch, das stzatliche Gewaltmonopol für die Zwecke der repressiven Universalisierung einer neuen Moral zu nutzen, birgt eine Reihe von Widersprüchen.

Die Rede von den Umwelusunden und den Umwelesündern wird von niemandem mehr metaphorisch verstanden. Aber ist dic Moral neu?... Nenn, neu ist diese Moral nichr, die aus der veränderen Wahrnehmung dor Umwelt folgt. Es ist eine des Verziches, der Disziplin, der Ordnung, des Gcseizes vor allem anderen.

Katharina Rucschky

Beispiel I: Umweltschurz oder Öko-Auroritarismus?

Die Polizei hac die Zeichen der Zeit erkannt und setzt hinsichtlich weiterer Expansionspläne inzwischen weniger auf den "Terrorismus* als auf den "Umweltschurz . In deutschen Großstädren bildete erst die Schurz-, dann auch die Kriminalpolizei während der siebziger Jahre kleire, aber sterig wachsende UmweirschuczGruppen. Die Zahl der Verfahren, aber auch die Höhe der verhängren Straien und die "Mithilfe der Bevölkerung" stieg rapide av. „Umweltschutz ist also eine neue und wirksame Art polizeilicher Selbstdarstellung “, resümierte ein Polizeihauptmeister aus Frankfurr im lokalen Hausblat (Loos 1978, 27). Da wollte die Kriminalpolizei nicht abseics stehen und gründece - 1985 - eine zehnköpfige „Umwelt-Kripo* aus sökologisch geschulten « Beamren. Durch die Verabschiedung des 18. Strafrechtsänderungsgesetzes von 1980 hatte man sich ermutigr gefühlt, in diesen Bereich zu investieren, kommende Gesetzesverschärfungen erhöhen die Attraktivität der Arbeir.

Die Staatsanwaltschaft, im Konkurrenzkampf der Instanzen um Macht- und Einflußvermehrung zweifellos einer der Gewinner des lerzten Jahrzehnts, kennt auch die für diesen Fall geeigneten Verbünderen. An die Adresse der »lieben Freunde « bei den Grünen schickre am 26. 1. 1984 ein hessischer Staatsanwalt auf dem Briefpapier des Bundesverbandes Umwelt und Nacurschuez (BUND) ein 24 seitiges Papier. Bei dem »mit solidarischen Grüßen weendeten Schreiben des Staatsanwalts handelete es sich um eine Ar Koalitionsangebot für einen Pakt zwischen Staatsanwaltschaft und Grünen, ausgearbeitet von einer vjustizinternen Arbeitsgruppe «, u. a. zum Themen- 
bereich »Bekämpfung der modernen Wirtschatrskriminalitäı (einschließlich Umweltkriminalirat) und ihre Lösungsmoglichkeiten. "Bei ihren Erörterungen über die *Lösungsmöglichkeıten $\alpha$, die - wie sollte es anders sein - vor allem in einer Ausdifferenzierung der Organisation und vielen, vielen neuen Planstellen gesehen wurden, hatre sich die Arbeizsgruppe winsb. von dem Grundgedanken leiren lassen, $\mathrm{d}_{2} \beta$ eine ökologische Wender nicht nur aul dem Papier, sondern nur in der prakrischen Politik durch Beseirigung des allenthalben anzutreffenden Vollzugsdefizits insbesondere im Bereich der Justiz- und Umweltschurzverwaltung criolgen muß. Eine besondere Bedeutung komme hierbei der Umwelt-Strafverfolgung zu und damir der Staatsanwalcschaft als , Herrin des Verfahrensa - angefeuert von einem breizen Presse- und einem wohlwollenden Basis-Echo konnten sich die Grünen im Landag diesen fortschrittsfreundlichen Kriminalisierungsagenten auch nichr verschließen (vgl. Frankfurter Rundschau v. 25. I. 1985; Brodersen/Kohl 1984, s).

In der Tat scheint dem Umweltschutz ein neues Band der Konsensstiftung gefunden, das von reches bis links reicht und den Unterschied zwischen den Parteien (endlich wieder?) verwischt. Da gibe es niche nur den (linken) „BUNDu, der für neue Kriminalisicrungen eincritt, sondern auch einen (rechten) "SCHUTZBUND der im "Deucschen Anzeiger", einer "Freiheitlichen Wochenzeirung am

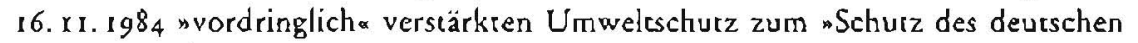
Waldes * norfalls auch ohne "Warten auf Entscheidungen der schwerfälligen. EGBürokranie" verlangte. Das klang noch harmlos erwa im Vergleich zum Vokabular, das die Berliner Alternative Liste wenig später ausgrub, als sie 2u Jahresbeginn 1985 immerhin schon von der "Vergifung der Böden und Pflanzen* als sden abscheulichsten Verbrechen « sprach und hiergegen narürlich "den norwendigen Niederschlag in der Gesetzgebung sowie in der sAusstatung und Ausbildung der Verfolgungsorgane forderte $(1985,279 \mathrm{f}$ ). Gibr es einen Grund für die Alternative Liste, die alles Lebende, Menschen, Tiere und Pflanzen davor schürzen will, nkrank gemacht und häufig dem Siechtum ausgesetzt zu werden (a. a. O.), der Forderung des (rechren) SCHUTZBUNDES (vom 30. I1. 1984) nicht zuzustimmen, die da lautere: Schucz auch ades ungeborenen Lebens, Kampí dern Abureibungsmißbrauch, großzügige Hilfe für Frauen und Familien *?

Und warum nicht auch Schurz vor Kriminaliät schlechthin oder - wieder in den Worten des SCHUTZBUNDES - härreres Durchgreifen gegen Kriminelle, die Eigenrum, Leib und Leben der Bürger bedrohen. Lebenslängliche $\mathrm{H}_{2}$ fı für Rauschgiftbosse $\alpha$ ?

Beispiel 2: Auf dem Weg zu einer grün-alternativen Zensurbehörde?

Die neuen sozialen Bewegungen erweisen sich mehr und mehr als ein Hort jener Arr von Reformern, die in der sozialwissenschafulichen Liceracur als Veranstalter nmoralischer Kreuzzüge (vgl. Treiber 1984) oder als "Unternehmer in Sachen Moral * (vgl. Becker 1973) bekannt und von der Relevanz ihres Zieles so fasziniert sind, daß ihnen die Mitzel zu dessen Erreichung nahezu gleichgülrig erscheinen. So kann es zum Beispiel passieren, daß Teilc der gesellschafichen Gruppierungen, die anläßlich der Sympathisancen-Jagd vor gerade einem Jahrzehnt noch heftig gegen die *Zensurparagraphen im Strafgesetzbuch opponiert hatren, dieselben Vorschrifien aus laurer(em) moralischen Eifer heute gutheißen. Der nguce Zweck - dic Bekämpfung von Horror-Videos - scheint viele Mirtel zu heiligen.

Die Grüne Parrei versteht sich als Repräsentanz der neuen Bewegungen. Deshalb läuft auch sie Gefahr, daß es ihr wie den neuen Moralunternehmern weniger um die 
Eignung des straigesezzlichen Mittels als um die symbolische Repräsentarion der nrichrigen Moral« gehr. Für eine Partei, die sich in Sachen Moral von niernanden übertreffen lassen will, stellen symbolische Geserzgebungsakce eine Dauerversuchung dar.

Nur um Haaresbreite sind die Grünen daran vorbeigekommen, ihre moralisch untadeligen Anliegen in einen Fall surafrechulicher Regulierung gesellschafdlicher Kommunikation umzumünzen. Um gegen faschistische Gruppen vorgehen zu können, die den nationalsozialistischen Judenmord leugnen und offendlich die Existenz von Gaskammern in deurschen Xonzentrationslagern in Abrede stellen ein "Kampfbund deurscher Soldaren a hatce die Unverfrorenheit, „für jede einwandfrei nachgewiesene , Vergasung in einer 'Gas-Kammer «x 10 000,- DM auszuloben-, hatre noch die sozialliberale Koalition eine Vorschrifr geplant, mir der die Lüge von der "Auschwitz-Lüge unter Srrafe gesteltr werden sollte. Die neue Bundesregierung hiele dem Encwurf ihrer Vorgängerin zunächst die Treue (BT-Ds 10/1286), verzichtete dann aber nach Kritik seitens der CSU und des Bundesrates in einem ersten Wende-Schritr auf die Kriminalisierung dex mündlichen Leugnung oder Verharmlosung nationalsozialistischer Gewaltverbrechen. Zumindesi am Stammrisch solite die Meinungsíreiheit voll erhalten bleiben (BT-Ds ro/r286, Anlagen 2 und 3). Aus derselben Richrung kam die Behauptung, es sei unbillig, die Leugnung der von den Deutschen begangenen Verbrechen zu bestrafen, die Leugnung der an den Deutschen begangenen Verbrechen - man war hier schnell mit dem Analogbegriff der "Verreibungslüge" bei der Hand - hingegen straffrei zu lassen. $D a$ die immer schon unklare Vorstellung darüber, welches Rechrsgur nun eigentlich geschürzt werden sollte, hinter der Nebelwand parteipolitischen Taktierens endgültig zu verschwinden drohre, wußse bald niemand mehe so rechr, wo der richrige Platz für die neue Vorschrift sei. Zuerst hatse die Änderung in $\$ Y_{4} 0 \mathrm{StGB}$ (Belohnung und Billigung von Straftaten), dann in einem neuzuschaffenden $\$$ rza (hinter Verherrlichung von Gewalt, Aufstachelung zum Rassenhaß) erfolgen sollen. Während der Justizminister zu Jahresbeginn $198 \mathrm{~s}$ für weitere Verwirrung sorgre, indem er sich mir großem Nachdruck für die Verabschiedung des 2 r. Strafrechtsänderungsgeserzes einserzre (ohne daß herauszufinden war, in welcher Fassung), wuchs der Koalitionsspizze die Konfusion über den Kopł. Noch im März 1985 handelte man die Rücknahme des Geseczentwurfs aus und beschloß einen Neuanfang. Der Justizminister sollte prüfen, ob man die (immer noch unklaren) Intentionen niche auch in den Beleidigungsdelikten der $\$$ \& $\&$; ff. StGB realisieren könne. Dort fand man dann schließlich eine Nische in der Bestimmung über den Strafantrag (\$ 194). Die Beleidigung, grundsärzlich nur auf Antrag hin verfolgr, wird - so vom Bundestag am 25.4. 1985 mit Mehrheis beschlossen - künftighin von Amts wegen verfolgt, - wenn der Verietzte als Angehöriger einer Gruppe unter der nacionalsozialistischen oder einer anderen Gewalt- und Willkürherrschaft verfolgt wurde, diese Gruppe Teil der Bevölkerung ist und die Beleidigung mit dieser Verfolgung zusammenhängt.»

Die strafgeserzliche Einschränkung der Meinungsfreiheit, die Vorverlagerung hoheitlich-repressiven Einschreitens bis weit in Sphäre gesellschaftlicher Kommunikarion hinein, war in Deurschland uradirionell ein Privileg der politischen Rechren, wenn man einmal von den diesbezüglichen Sündenfällen der sozialliberalen AntiTerror-Gesetzgebung absieht. Es steht allerdings zu befürchten, daß die (relative) Tugendhafrigkeir des progressiven Lagers auch nur auf einem Mangel an Gelegenheit beruhte. Der Konflikt um das 21. Strafrechtsänderungsgeserz zeigt denn auch bei denjenigen, die dem Geserz schließlich nicht zustimmen, erhebliche Einbruchsstellen für Bestrafungsgelüste. Weder die SPD noch die Grünen konnten sich für 
eine prinzipiell restriktive, $d . h$. die Kommunikationssphäre von strafrechticher Intervention freihaltende Handhabung des Kriminalrechts entscheiden. Die SPD lehnte den Regierungsentwurf zwar ab, hielt aber an ihrem ursprünglichen Plan zu $\$ 140$ StGB fest (BT-Ds 10/32 66 v. 24. 4. 1985). Die Grünen ähnelten sich dieser Posirion an, wollten jedoch den Weg über eine Änderung des $\$ 194$ II StGB wählen (BT-Ds $10 / 3255$ v. 24.4.1985). Ein Gutachten für die Grüne Bundescagsfraktion erklärte das Rechesgur so: „Alle Lebenden, die unmitrelbar oder mittelbar von der Nazi-Herrschaft Gewalt und Terror erfahren haben, sollen in dem Wabrhertsanspruch ibres erlittenen Lesdens, in ibrer geschuchclichen Identität geschützt werden. " ${ }^{1}$ Der riskanten Zuversicht, die Strafjustiz sei der geeignete Hürer des unverfälschten Geschichrsbildes, entsprach der gleichsam antisoziologische Glaube, durch die Errichung polizeilich kontrollierter Grenzen legitimer politischer Diskussion »ejner Wiederholung der nationalsozialistischen Diktacur vorzubeugen " (Ostendorf 1985, 1062). Auf eines der bedenklichsten Gesetzesvorhaben seir dem Kontaktsperregeserz (vgl. im einzelnen Cobler 1985) vermochte nicht nur die chronisch etatistische SPD, sondern vermochten auch die parlamentarischen Repräsentanten der neuen sozialen Bewegungen keine überzeugende Antwort zu finden.

Eine andere, frischer sprudcinde Quelle von Moral wird seil Anfang der siebziger Jahre von den frauen betrieben.

Katharina Rutschky

Beispiel 3: Moralunternehmerinnen

Aul die Frage, warum sich die ehemals libertär orientierte italienische Frauenbewegung Ende der siebziger Jahre ausgerechnet hincer dem Vorschlag einer Gruppe von Juristinnen zur (schärferen) Neufassung des Gesetzes gegen sexuelle Gewalt rviederfand, suchte Tamar Picch (1983) eine Anrworn.

Thre These: Die ökonomische und politische Krise hatre die Mobilisierungsfähigkeit der Bewegung geschwächt, so daß eine Rücknahme der emanzipatorischen zugunsten von parrizipatorischen Forderungen und von materieller auf formelle und prozedurale Rechrsgleichheir nahelag. Der iralienische Geserzentwurf spaßren in die Systemarik der Strafrechisdogmatik, er perfektionierte sie sogar, indem er die Rechrsgleichheir von Mann und Frau betonte, während weicergehende Kritik am Strafrecht im Zusammenhang mit dieser Karnpagne ausgeblendet wurde. Auf die Frage, ob sich die Juriscinnen von dem Gesecx längere Scráen für Vergewalrigungen und/oder einen besseren Schutz der Frauen versprachen, erhielt Pitch typischerweise zur Antwort: ( $r$ ) Wir wollen gar nicht unbeding längere Strafen für Vergewaluiger, wir wollen dieselbe Gerechtigkeit, die andere Verbrechensopfer auch erhalten; (2) Das Strafrecht ist sowieso smännlich«. Wir sind dafür nicht verantwortich. Wir wollen nur, daß es nach seiner eigenen Logik konsistent angewands wird: (3) Wir sind an den Details des Entwurfs gar nicht so interessiert. Wir wollen nur, daß ein paar wesendiche Prinzipien - z. B. die Würde der Frau und die Anerkennung von Gewalt gegen Frauen als schweres Verbrechen - klargestellt werden; (4) Wir wissen, daß ein neues Gesecz weder die Verbrechensrate senken

I Hervorhcbung nicht im Ongual; vgl. hierzu die Rechesguebestımmung durch den Jusezmuster: Dic Leugnung der Beschönigung von NS-Verbrechen sei greignet, das $\rightarrow$ RechesbewuBisein der Bevolkerung zu kränken.e Ist der Anspruch des Bügers auf en -ungekränktes RechesbewuBrsein. etst einmal anerkanne, steht der Mulupliksuon von Rechesgitem und der Oberwindung aller herkömmlichen Grenzen staatichen Surafens nachis mehr im Wege. 
noch über längere Frciheitsstrafen irgendeine grundsätzliche Veränderung der Problematik zu erreichen sein wird. Der Enrwurf ist aber immerhin eme Gelegenheic, die öffentliche Sensibilicäı für diese Fragen zu erhöhen.

In Deutschland waren die siebziger Jahre vom Kampl gegen den Abtreibungsparagraphen bescimmr. Im Juni 197 r hatte die Illustrierte "Stern * die Selbstbezichtigung von 374 Frauen ( $\$$ Ich habe abgetrieben*) veröffenclicht und damir eine Kampagne der Frauenbewegung stark gemacht, ohne die es kaum zur Fristenlösung gekommen wäre (vgl. s. Strafrechtsreformgeserz v. 2.3. 1974, BGBl. I, 469), die dann allerdings vom Verfassungsgericht verhindert wurde (Urt. v. 25. 2. 1975, JZ 1975, 205). Spätere Strafrechisprojekte richteren sich nicht auf $\mathrm{Ab}$-, sondern Neuschaffung. Im Juli 1983 begrüßte die vrageszeirung * den Vorstoß der Hamburger Juscizsenatorin, über den Bundestat eine Gesetzesinitiative einzubringen, die den Vergewaltigungstatbestand - nach $\$$ I 77 StGB beschränkt auf außereheliche i Beischlaf \&-Nötigung - auf eheliche Nörigung ausdehnen solice. Kommentar der (Hamburger) Grün-Altemativen Liste, warum sie niche selbst einen Geserzentwuri vorgelegt habe: "Da har unser Frauenbereich geschlafen. - - Vermuclicha, so die staz *, würden "Notruf-Frauen, alternative und grüne Parlamentarier zur Kennınis nehmen müssen, daß ein Teil ihrer ureigensten Basisakrivitäten (...) von den ausgeschlafenen SPDlern überrommen worden ist. " In der Kommentar-Spalce derselben Zeitung wird unter der Überschrift •Ein Gesetz, das Frauen schützr kargestellr, daß frau sehr wohl um die begrenzte generalpräventive Wirksamkeit von Strafgesetzen wisse: "Selbstverständlichu würden »durch ein Gesecz « die Vergewaltigungen in der Ehe wzunächsc nicht weniger . Darum gehe es aber auch gar nicht so sehr, der Vorteil liege vielmehr in der öffendichen Anerkennung des Unrechts, das den verheirateten Frauen angetan wird. Die strafrechtiche Verankerung des Tatbestands sollte dazu dienen, "dies in der Ölfentlichkeit $\alpha$, svor einem Gerich $\alpha$ zu thematisieren. Entsprechende Taten würden dadurch nicht unmitrelbar reduzierc, aber wie rverden durch die Benennung als Straftatbestand zunächst einmal als vorhanden und strafwürdig bezeichnet". Die Geserzgebungsinitiative kann nicht nur deshalb als symbolische im Sinne Edelmans (1976) bezeichnet werden, weil sie - den Iniriaroren bewußc - von vomherein an den Kräfceverhältnissen im Parlament scheicern mußre, sondern deswegen, weil das Gesetz, selbst wenn es zustandegekommen wäre, gar nicht den Zweck gehabc hätre, in einem instrumentellen Sinne implemenciert zu werden: "Selbst wenn diese Gesetzesinitiative der Hamburger SPD zur Zeic nicht viel Aussicht hat, durchgeserzt zu werden, selbst wenn diese Geserzesvorlage zu vorsichrig und ängstlich formulier ist, selbst wenn die SPD dami versucht, takrisch Wahlerinnen zu gewinnen, - allein, daß das Thema mal wieder als Problem öffentlich gemache wird, lohnt solche Iniziativen" (taz v. 26.7. 1983).

Die (Berliner) Alsernative Liste fordert mir der Frauenbewegung in ihrem Wahlprogramm rg8s mehr: außer der Streichung des Wortes saußerehelich" in den $\$ 977$, $178 \mathrm{StGB}$ und der "Aufhebung der sminder schweren Fällee ( $\$$ I 77 Abs. 2 bestraft die Vergewaltigung in minder schweren Fallen mit Freiheitsentzug von sechs Monacen bis zu fünf Jahren) auch peine Neudefinition des Vergewaltigungsbegriffs, nach der jede Form von oraler und analer der vaginalen Penetration gleichgestellc ist. Die Begrifflichkeic serzwungener Beischlafe muß geänder werden in sgegen den Willenı. Daneben soll nicht nur die Justizorganisation verändere werden ("Schaffung einer Spezialabteilung an jedem Gericht, dic sich ausschlicßlich mit Straftaten gegen die sexuelle Selbstbestimmung von Fraven und Mädchen befaßt, wobei diese Abreilung ausschließlich mir Fraven zu beserzen is( $\alpha$ ), sondern auch die Einstellung der Beschäfrigren win den bestehenden Inscirutionen ", und zwar durch "Maßnahmen zur Sensibilisienug* (Alternacive Liste 1985,21 f.). 
Auch Strafvorschriften, die - wie die $\$ \$ 173$ und 176 StGB - in ihrer Schurzwirkung

höchst umstritten sind und möglicherweise mehr schaden als mutzen (vgl. Jung 1983), werden im Zuge des neuen Trends zur Moralgesetzgebung wiederbclebr. Alle Übergriffe auf Mädchen, auch wheimliche, vorsichtige Berührungen* sollen strafbar bleiben oder werden (vgl. Kavemann, Lohstörer 1984 , 10). Während die Justizkricik der sechziger Jahre nachdrücklich auf das häufige Mißverhältnis zwischen einer (relativ gut verarbciteten) sexuellen Beläsrigung und einer (nichr setten traumatisierenden) Belästigung durch die Vernehmung im Rahmen der Justizprozeduren andererseits hinwies, spiejt dieser Gesichtspunkt in dem Moralkreuzzug, der » Väter als Tater brandmarken solk, kaum noch eine Rolle. Wichtig ist allein das Ziel, weniger die Wahl des Mitzels. Auf die Frage, ob sich denn der Weg durch die Männerjustiz lohne, wird die Hinwendung zu den Instanzen der sozialen Kontrolle •grundsäılich befürwortet, sei sie doch of wder einzig wirksame Schucz, denn selbst wenn ihr Verhalcen aufgedeckt wurde, finden Männer immer Wege, die Übergriffe fortzusetzen, wenn nicht der Druck eines laufenden Ermittlungsverfahrens besteht " (Kavemann/Lohstöter 1984, 122).

In jedem Moralunternehmen geht es nicht nur um professionelle Vorteile (z. B. für Anwätinnen), sondern auch um die Reorganisation des Weltbildes in ein "gutesa und ein wböses" Lager, wobei dialektische Betrachtungen abgewehrt werden. Die „Täter müssen nböse«, die „Opfer* müssen nunschuldig " sein (vgl. Christie r984). Bei Moraldelikten (lïsterner Blick, vorsichtige Berührung) kommt das Problem der Beweisbarkeir hinzu. Moralunternehmer beziehen da gerne klare Stellung. Dies geht z. B. aus einem Interview zur Frage des sexuellen Kindesmißbrauchs mit einem Gerichtsgutachter hervor. „Dieses Verbrechen", so die Interviewerin, müsse doch alle Männer angehen. Der Gucachter verweist auf erste, allerdings "ganz geringe“ Erweiterungen »des diesbezüglichen Bewußseins bei Männernæ, das snur durch intensive Aufklärung unter Vermeidung unsachlicher Übertreibung weiter enrwikkelt werden könne. Auf die Nachfrage, was er denn wohl unter wunsachlichen Übercreibungen « verstehe, erklärt der Gutachter, solche bestünden z. B. darin, daß ndie Ursachen solchen Täterverhaltens nur als Bösartigkeit* angesehen würden. ¿Dabei wird übersehen, daß die Tàter unter Umsränden auch Opfer sein können; Opfer ihrer eigenen falschen Erziehung, ihrer eigenen falschen Vorstellungen und ihrer gestörten Bedürfnishaltung. Man kommt in diesem Bereich nur weiter, wenn man versucht, sowohl den Tätern als auch den Opfern vorurteilslos zu begegnen und sie zusammenzubringen. « Darauf die Interviewerin: "Das klinge für mich nach Entschuldigung der Tärer." Sie frage dann nach den Gründen für die geringe Verurteilungsquote bei diesen Delikten, was vom Gutachter mit den Bewcisschwierigkeiten und mit Problemen der Zurechnungsfähigkeit beantwortet wird. Darauf die Interviewerin erstaunt: was heißr, einem Tàter wurde nicht mit der nörigen Sicherheit die Tat nachgewiesen? Dabei geht es doch darum, ob dem Opfer, der Zeugin, geglaubr wurde oder nicht $\propto$ (G. Hentschel, taz 30. Ir. I984).

Die sters gefährdere Balance des rechtsstaatlichen Strafverfahrens wird von sozialen Bewegungen in Mitleidenschaft gezogen, doch die Verfahrensgrundsätze, die im Interesse einer Gruppe ausgehöhlt werden, können sich bei nächster Gelegenheir als dringend erforderlich (aber dann nicht mehr vorhanden) erweisen. Die amerikanische Feministin Susan Brorvnmiller begrüßre z. B. jüngst die Fernsehüberragung aus dem Gerichrssaal, in dem über eine spektakuläre Vergewal cigung verhandelt wurde, da sie meinte, die Übertragung werde mehr Frauen ermurigen, Vergewaligungen anzuzeigen. Was zu sehen war - die Aussage des Opfers, die Befragung durch Anklage und Verteidigung - konnte freilich theorerisch auch für manche Zuschauer eine Arr Abendunterhaliung darstellen, vergleichbar einem Horror-Video. 
An dieser Srelle drängen sich - abgesehen von der Notwendigkeir, dic skizzierte Enrwicklung empirisch zu fundieren - weitere Fragen auf: Welche Rolle spielt die ökonomische Krise und welche die politische Krise der Linken bei der Hervorbringung des etatistischen Umschlags? Ist die Flucht in die Moralgeserzgebung nicht vielleicht auch ein charakteristischer Ausdruck für das Identizärsdilemma ciner parlamentarisierten Bewegung, weil sie als "Polirik durch Symbole* noch eine gewisse Wahlverwandischaft zu außerparlamentarischen Politikformen aufweist und zugleich Agieren in den Institutionen ermöglicht, ohne zur Absorprion durch bürokratische Detailarbeit zu führen? Schließlich: Bestätigt sich hier nicht - unserem Sk eprizismus gegenüber den immer leichr ahistorisch erscheinenden Ablauimodellen für soziale Bewegungen zum Trorz - die Unhintergehbarkeic der Institutionalisierung aller sozialen Bewegungen bei Srrafe des Untergangs (vgl. KarstedtHenke ig80)? Weirerhin könnte man aber auch der Vermutung von Zusammenhängen zrvischen dem institutionelien Umschiag der neuen sozialen Bewegungen und bestımmzen Veränderungen in der kriminologischen Wissensproduktion nachgehen. Dies soll im folgenden skizzier werden.

Leitfigur und Funktionalität. Mit der Vorsteliung einer historischen Bedeutung der kulturrevolutionären studentischen Avantgarde (Marcuse) bzw. der morganischen Intellekruellen (Gramsci) har die (klein-)bürgerliche Intelligenz unter anderem ihr eigenes Identicätsproblem - das der policischen und sozialen Heimaclosigkeit einer "Zwischenklasse * (Quensel 1984, 204) von "Advokaten ohne Klientenauftrag* (Giesen 1983,233 ) - zu lösen versucht. Und fraglos beruht ihre Actrakcivität in der Neuen Linken unter anderem auch darauf, daß diese Konzeptionen dem Intellekruellen ein angenehmeres Selbstbild vermitceln als etwa die Vorstellung, obnmächriges Objekr einer undurchschauren Funktionalitäc oder gar ein "stiller Teilhaber von Moralkreuzzügen (Brodersen 1984) zu sein. Wo aber ist die Neue Kriminologie auf dem Koncinuum zwischen Sub- und Objekt anzusiedeln?

Die junge, neue, kritische und radikale Kriminologie ist aus dem Kontext der Neuen Linken entstanden und hat sich - in gedämpitem Ton, wie es sich für Wissenschaft gehört - auf deren Leitshemen bezogen. Vereinfachs wird man darüberhinaus feststellen können, daß beide - die Neue Linke und die Neue Kriminologie - ecwa in dem Maße enfolgreich waren, als ihre Vorstellungen sich für die Modernisierung des Systems der Sozialkoncrolle als brauchbar erwiesen. Die Neue Kriminologie war insofern doppelt funkrional: Nicht nur vermochte sie die von der Neuen Linken themarisierten Strafrechrskririken wissenschaftlich erwas abzusichern, ihr (begrenzter) Erfolg war darüberhinaus auch deswegen möglich, weil sich auf der Ebene der Hauptgeselischaft weine Verschiebung in der widersprüchlichen Dynamik von Ausgrenzung und Integration in Richnong auf mehr Integration als notwendig herausgestell hatte: Wer die Fähigkeir zu arbeiren und zu konsumieren hatre, sollre das auch tun können, und - andersherum gesehen - niemand, der diese Fähigkeit hatre, sollte disqualifiziert werden (...), und auch die Reformen auf den Gebieten der Sozialarbeit, des Suralrechis und des Strafvollzugs (...) entsprachen diesem Bemühen, niemanden auszugrenzena (Steinert 1984,87 ).

Dieser Phase entsprach die Infragestellung der symbolischen Funkrionen des Srrafrechts und seine Neukonzeprion als bloßes Instrument der Konfliktregelung (vgl. Steinert $198 \mathrm{~s}$ ). Es war die große Zeit der negativen Kritik und theoretischer Ansätze, die - wie das interpretative Paradigma - im wesentichen entlegitimierend wirken konnten, weil sie die Bedingtheit und Konstruiertheit von *Norm* und "Abweichung", die Unhalrbarkeit von "Tärertypen* usw. nachhairig vor Augen 
führten. Aus diesen Gründen, so dürfen wir vermuten, rührte auch die einstige Popularität der Moralunternehmer-Forschung.

In dem Maße, in dem sich die politische Bezugs-Kuleur der Neuen Kriminologie transformierte, taten dies auch ihre Forschungsthemen und-zugänge. Organischer Intellektueller und/oder suller Teilhaber? In der antiautoritären Phase der Neuen Linken war auch die Neue Kriminologie anciautoritär - sie kritisierte die Polizei und die Gerichte, problematisierte den staatlichen Strafanspruch und insbesondere die Verquickung von Strafrechr und Moral. Das Strafrecht, so der Tenor der Kririk, sei nicht dazu da, moralische Werte hochzuhalten. Als Ausdruck des Machrverhälınisses gegnerischer Klassen betrachrere man es mit großer Skepsis. Der Erikertierungsansaez abweichenden Verhalteos (vgl. Sack 1972) wurde dementsprechend von den Praktikern aus den Instanzen der Sozialkontrolle dafür gescholten, daß er für sie - nichrs bringi $\times$. Und dies war seine Absicht. Der Topos des moralischen Kreuzzugs war der theoretisch geronnene Ausdruck dieser kricischen Distanz zu Staat und Recht.

Doch mir dem für die wissenschaftliche Produktion typischen *time lag* fand auch die Transformation der neuen sozialen Bewegungen ihren Weg in die Neue Kriminologie. Die Efforschung der $n$ Kriminalizät der Mächtigen« drückte einerseits (negarive) Kritik im Sinne des Topos der "Klassenjustiz a aus, konnte aber andererseics auch schon als Aufforderung zum (posiciven) Handein, nämlich zur effektiveren Verfolgung statushoher Personen, im Sinne angeblicher sozialkompensatorischer Funkrionen des Strafrechts verstanden werden (vgl. Pfeiffer/Scheerer r979). Eine ähnliche Ambivalen` besaß die Normgenese-Forschung: Einerseits in Weiterführung der Labeling-Perspektive nur (negativ) auf die Partikularinteressen hinter Normen mit Unversalitätsanspruch aufmerksam machend, zeigze sie Anfang der acheziger Jahre ein überraschend posicives Potential.

Plötzlich ging es um Gesetze, deren Inhalte den Werten der neuen sozialen Bewegungen entsprachen, insbesondere um Umwelr-Strafgeserze. Allem Anschein nach bahne sich eine Wandlung vom Kritiker zum Zulieferer oder sillen Teilhaber von Moralkreuzzügen an (Beispiele bei Brodersen/Kohl $19 \$_{4}$ ). Der veränderten Funktion würde die Verabschiedung des interprecativen Paradigmas zugunsten von Theorieansärzen, die sich der ontologisierenden Sichrweise moralischer Unrernehmer (z. B. ihren Tätertypologien: "der Umweitstrafiäter», oder Vergewaltiger» usw.) gegenüber aufgeschlossener zeigen, entsprechen. Als Theorieansätze mit einer konsirukivistisch handhabbaren sozialtechnologischen Axiomatik bieren sich gegenwärtig der nNew Realism* in seinen verschiedenen Spielarten (z. B. Taylor 198 1 ) und ein wnaiver Realismus» (der sich selbsc als "realen Abolitionismus* bezeichner, vgl. Haferkamp 1984) an. Beide sind aufgrund ihrer unhinterfragien Übernahme von Ideologemen wie "Kriminalität $\alpha$, der Täter-Opfer-Dichotomie usw. geeignet, im Namen fortschritrlicher Kriminalpolicik zu Ausbesserungsarbeicen am löchrigen Flickenteppich der Legitimarion scaarlichen Strafens herangezogen zu werden. Indem die neuen sozialen Bewegungen und ihre stillen Teilhaber die symbolischen Funktionsbereiche des Strafrechts emphatisch bekräfrigen, tendieren sie zu einem systemcheorecisch fundierten Verscändrus des Strafrechts, das dessen Hauptiunktion in der symbolisch vermitzelten Sicherung des Rechtsvertrauens und darüber vermiztelt des Gesamtsysterns sieht. Einer Wiedervereinigung der kritischen Kriminologie mit der herrschenden Lehre einer systemtheorecischen Neubegründung der Strafe (vgl. Baratta 1984) stehen dann nur noch der Labeling Approach und der Abolitionismus im Wege. 
Alternative Liste, Hg., Wahlprogramm der Altemativen Listc. Berlin 1985.

Baratra, A., Integration - Präventon. Eine systemeheoreusche Neubegründung der Surafe. KrimJ 16. 1984,1$\} 2-148$.

Becker, H.S., Außensciter. Zur Soziologie abweichenden Verhaliens. Fim 1973.

Brodersen, C.N., Moralunternehmer und stille Teilhaber. Das Andere Transparent (DAT) \#* 9/Sept. $1984,18-21$.

Brodersen, C.N., B. Kohl, Moralunternehmen - eine Wachstumsbranche? Das Andere Transparene (DAT) \# 10/Dez. $1984,4-8$.

Chruste, N., Sociery and the Vicum. Manuskript Oslo 1983.

Cobler, S., Das Gesezz gegen die ouschwizz-Lügex. KJ 18, 1985, 159-170.

Edelmann, M., Politik als Rirual. Frankfurd New York 1976.

Giesen, B., Moralische Unternehmer und öffendiche Diskussion. Überlegungen zur gesellschafdichen Thematisıerung sozialer Probleme. Kölner Zeicschrifu für Soziologe und Sozıalpsychologie $35.198 ; 1230-254$.

Haferkamp, H., Herrschaftsverlust und Sankrionsverziche KrimJ 16. 1984, 112-13 I.

Heldmann, H.-H., wAuswirkungen auf das algemenc Preisniveau sind nichi zu erwarena, Konkres 6/1984,45-47.

Karstede-Henke, S., Soziale Bewegung und Terrorismus, in: E. Blankenburg, Hg., Politik der inneren Sicherhett. Frankfurr a. M. 1980, S. 159-221.

Kavemann, B., L. Lobstöter, Väter als Tatcr. Sexuelle Gewalt gegen Mädchen. Reunbek 1984. Loos, P., Umwelsschurz enne Aufgabe der Polizei? Ein Bericht über die Tärigkeit der Zenuralen Umwelschurzgruppe der Frankfurer Schurzpolizei. In: Polizei in Frankfurr '78, 23-31.

Ostendorf, H., Im Streit: Die strafrechtliche Verfolgung der suschwitz-Lüge. NJW 1985, $1062-1063$.

Pfeiffer, D.K., S. Scheezer, Kriminalsoziologie. Surtgart 1979.

Pitcb, T. Critical Criminology. The Construcion of Social Problems and the Question of Rape. Unv. Manuskeript Perugla 1983.

Quensel, St., Kriminologische Forschung: Für wen? Oder: Grenzen eıner rauonalen Krimınalpolitik, Knm\} I6. 1984, 201-217.

Roth, R., Neue sozale Bewegungen. Literanur Rundschau. Sonderhefe Neue Praxis Heft $7^{\prime} 1982,79-94$.

Rutscbky, K., Die Lust an der Selbstdarstellung. FAZ v. 22. 12. 1984 (Bilder und Zeiter).

Sack, $F$., Definition von Kriminalitäl als policisches Handeln: der labeling approach. KrimJ 4. [972, 3-j].

Steiners, H., Was ist eigentlich aus der "Neuen Kriminologıe geworden? Einige Thesen, um die Suche zu orienveren. KnmJ 16. $1984,86-89$.

Stemert, H., Alternadiven zum Strafrecht. In: G. K.iser u. a., Hg., Kleines Kruminologisches Wörterbuch. Heidelberg $1985^{4}, 9-14$.

Taylor, 1, Law and Order: Arguments for Sacialism. London 1981.

Treiber, $H$., Die gesellschafrliche Auseınandersetzung mit dem Terrorismus: Die Inszenıerung symbolischer Kreuzzuger zur Darstellung von Bedrohungen der normauven Orónung von Gesellschafi und Staas. In: F. Sack, H. Steıner, Protest und Reakcion. Oplader 1984, $320-365$. 